Markus Loew

Sektion für die Schulter- und Ellenbogenchirurgie, Stiftung Orthopädische Universitätsklinik Heidelberg

\title{
Differenzierte Operationsindikation bei posttraumatischer Ellbogenkontraktur
}

\section{Zusammenfassung}

Die posttraumatische Kontraktur des Ellbogengelenks ist Symptom verschiedener lokaler Veränderungen und systemischer Störungen.Systematisch werden intrinsische Kontrakturen, die von der Synovialis und intraartikulären Strukturen ausgehen, von extrinsischen Kontrakturen, die die Gelenkkapsel und extraartikuläre Weichteile betreffen, unterschieden. Ätiologisch ungeklärt nehmen die ektopen Ossifikationen eine Sonderstellung ein. Klinisch erfolgt die Klassifikation der Kontraktur nach dem Ausmaß der Restbeweglichkeit. Eine Operationsindikation ergibt sich bei hohem Schweregrad mit Schmerzen und funktioneller Beeinträchtigung. Die chirurgische Arthrolyse umfasst ein weites Spektrum von geschlossenen Verfahren, endoskopischen und offenen Operationen bis zur Totalendoprothese. Die operativen Maßnahmen sind relativ komplikationsträchtig, der individuelle Funktionsgewinn ist nicht sicher vorhersehbar.

\section{Schlüsselwörter}

Kontraktur · Ellbogen - Ossifikationen · Arthrolyse ie Bewegungseinschränkung ist ein uniformes Symptom äußerst heterogener Erkrankungen des Ellbogengelenks. Sie beruht auf unterschiedlichen pathophysiologischen Mechanismen, die den Spontanverlauf, die Behandlung und die Prognose entscheidend bestimmen.

Posttraumatische Kontrakturen betreffen häufig jüngere, aktive Patienten mit hohem Anspruch an die Beweglichkeit. Die Bewegungseinschränkung kann sowohl die Flexions-Extensions-Bewegung als auch die Pro- und Supination isoliert oder kombiniert betreffen. Ein Beuge- oder Streckdefizit bei freier Umwendebewegung ist häufig, während umgekehrt die Pro- und Supination nur selten isoliert eingeschränkt sind. Das Ausmaß der funktionellen Beeinträchtigung ist einerseits von der Schwere der Kontraktur und von ihrer Schmerzhaftigkeit, andererseits auch von der Beweglichkeit der benachbarten Schulterund Handgelenke abhängig.

Die Behandlung der Ellbogenkontraktur setzt die differenzierte Kenntnis der Ätiologie und Pathogenese, der betroffenen anatomischen Strukturen sowie eine kritische Analyse der individuellen Betroffenheit und der zu erwartenden Resultate konservativer und operativer Maßnahmen voraus [6].

\section{Klassifikation}

In Abhängigkeit von der Mobilitätseinschränkung ist eine Einteilung der Ellbogenkontraktur in verschiedene Schweregrade gebräuchlich [11], die sich ausschließlich am Gesamtausmaß der ver-

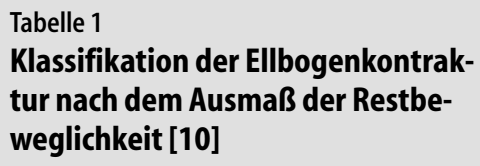

Tabelle 1

Klassifikation der Ellbogenkontraktur nach dem Ausmaß der Restbeweglichkeit [10]

\begin{tabular}{lc} 
Schweregrad & Gesamtflexion \\
\hline Gering & $>90^{\circ}$ \\
Mäßig & $60-90^{\circ}$ \\
Schwer & $30-60^{\circ}$ \\
Sehr schwer & $<30^{\circ}$
\end{tabular}

bleibenden Flexions-Extensions-Bewegung (Flexionsbogen) orientieren (Tabelle 1). Diese Klassifikation vernachlässigt die Umwendebewegungen und gibt dadurch die funktionelle Beeinträchtigung nur unvollständig wieder. Zudem ist es von Bedeutung, in welchem Winkelbereich der freie Flexionsbogen liegt. Morrey u. Kai-Nan [11] definierten den „funktionellen Bogen“ in einem Bewegungsbereich von $0-30-130^{\circ}$ nach der Normalnullmethode; eine weiterer Verlust an Flexion führt zu einem stärkeren funktionellen Defizit als die Verschlechterung der Streckfähigkeit, da bei geringerer Beugung auch mit Kompensations-

Prof. Dr. Markus Loew

Sektion für die Schulter- und

Ellenbogenchirurgie, Stiftung Orthopädische Universitätsklinik Heidelberg, Schlierbacher Landstraße 200a, 69118 Heidelberg,

E-Mail:markus.loew@ok.uni-heidelberg.de, Tel.:06221-966224, Fax:06221-968388 


\section{Loew \\ Indications for surgery in posttraumatic contracture of the elbow}

\begin{abstract}
Posttraumatic contracture of the elbow joint is a symptom that can be caused by various local changes and systemic disorders. Appropriate therapy can only be given if the operator is familiar with the relevant anatomical structures and the etiology and pathogenesis of the functional restriction are known. Intrinsic contractures arising from the synovial membrane and intraarticular structures must be systematically distinguished from extrinsic contractures affecting extraarticular soft tissues. The etiology of heterotopic ossifications is still widely unknown. Contractures are graded clinically by the residual range of motion. Surgery is indicated in severe cases with pain and loss of function. Surgical arthrolysis spans a wide spectrum ranging from endocopical procedures through open procedures to prosthetic joint replacement. Complications are relatively frequent in operations on the elbow, and the individual gain in function is not always predictable.
\end{abstract}

\section{Keywords}

Contracture · Elbow · Ossification . Arthrolysis

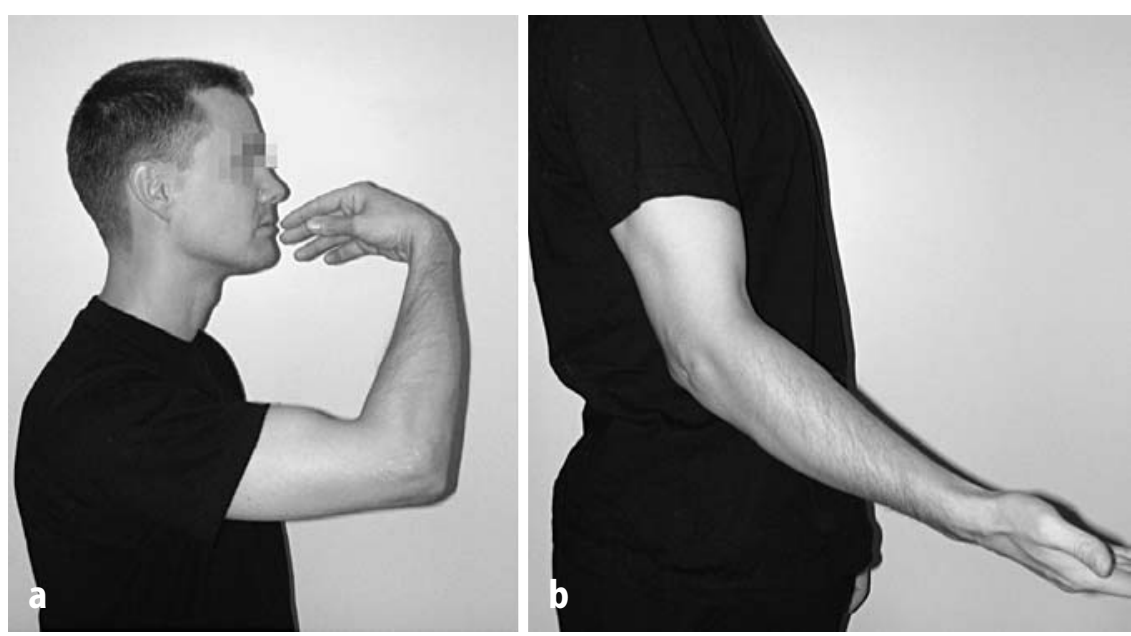

Abb. 1a,b $\Delta$ Mäßiggradige Ellbogenkontraktur (Extension-Flexion: 0-40-90 ${ }^{\circ}$ ) mit erheblicher funktioneller Beeinträchtigung

bewegungen im Schultergelenk die Hand nicht zum Mund geführt werden kann.

Nach ihrem pathomorphologischen Substrat können intrinsische von extrinsischen Kontrakturen unterschieden werden. Bei einer intrinsischen Ellbogensteife liegt die Ursache intraartikulär und betrifft die Gelenkfläche, den Knorpel oder die Gelenkinnenhaut; bei der extrinsischen Kontraktur führen Veränderungen extraartikulärer Strukturen wie von Kapsel, Muskulatur, Sehnen, Knochen und Haut zur Bewegungseinschränkung. Dabei ist es möglich, dass eine primär intrinsische Schädigung, z. B. eine Knorpelläsion, sekundär durch die Entwicklung einer Kapselfibrose zu einer extrinsischen Kontraktur führt.

\section{Prinzipien der operativen Behandlung}

Die Indikation zu einer operativen Behandlung der posttraumatischen Ellbogensteife richtet sich nach der Schmerzhaftigkeit der Bewegungseinschränkung, der funktionellen Beeinträchtigung und dem zu erwartenden Spontanverlauf.

Bei $>6$ Monate fortbestehender therapieresistenter Bewegungseinschränkung sind in Abhängigkeit von der funktionellen Beeinträchtigung operative Maßnahmen in Erwägung zu ziehen [1, 4, 7, 15]. Die chirurgische Arthrolyse ist auch bei einer einseitigen Streckhemmung $>60^{\circ}$ und einer Beugefähigkeit $<100^{\circ}$ indiziert (Abb. 1). Zwar kann auch eine geringere Streckhemmung bei
Schwerarbeitern oder Streichmusikern $\mathrm{zu}$ einer nicht tolerierbaren funktionellen Beeinträchtigung führen, ein Streckdefizit $<30^{\circ}$ stellt jedoch nur im Ausnahmefall eine Indikation zu einem bewegungsverbessernden Eingriff dar.

Bei der Indikationsstellung zu bewegungsverbessernden Eingriffen am Ellbogengelenk ist die relativ hohe Komplikationsrate der verschiedenen Operationen zu berücksichtigen. Diese ist v. a. auf die unmittelbare Nachbarschaft neurovaskulärer Strukturen zur Gelenkkapsel und zu den chirurgischen und endoskopischen Zugangswegen zurückzuführen. Bei der arthroskopischen Arthrolyse bedingen die Enge und die Kongruenz des Gelenkspalts die Gefahr iatrogener Knorpelläsionen. Transitorische Nervenirritationen wurden in etwa $5 \%$ der Fälle beschrieben [12]. Auch bei dem weichteilschonenden lateralen Zugang zur offenen chirurgischen Arthrolyse kommt es in $10 \%$ der Fälle zu einer meist vorübergehenden Dysfunktion des N. ulnaris und des N. radialis. Intraartikuläre Blutungen können wiederum die Entwicklung sekundärer Ossifikationen verursachen.

\section{Intrinsische Kontrakturen}

Die pathomorphologische Ursache intrinsischer Kontrakturen ist in der Veränderung von Gelenkbinnenstrukturen zu finden.

Bereits bei unverschobenen oder gering dislozierten Gelenkfrakturen kann der Hämarthros über Hämosiderinein- 
lagerungen in die Synovialmembran zu einer Kapselfibrose führen. Typische Folge von Frakturen mit Gelenkflächenbeteiligung im Wachstums- und Erwachsenenalter sind persistierende Stufenbildung und pathologische Kongruenz der Gelenkpartner. Die Folge von Radiusköpfchenfrakturen mit Depression oder Dislokation der Gelenkflächen ist eine Inkongruenz im proximalen Radioulnargelenk mit häufig schmerzhafter Einschränkung der Pro- und Supinationsbewegung und sekundärer Einengung des Flexionsbogens. Dislozierte osteochondrale Fragmente (traumatische Osteochondrosis dissecans) können zu Einklemmungen, Knorpeldestruktionen und zu einer progredienten Bewegungseinschränkung führen. Nur selten sind bei drohenden intrinsischen Kontrakturen präventive operative Eingriffe zur Vermeidung fortschreitender destruktiver Gelenkschäden indiziert. Die frühzeitige Punktion eines Hämarthros kann möglicherweise die Häufigkeit und Ausprägung einer posttraumatischen Kapselfibrose ebenso vermindern wie die endoskopische Entfernung freier $\mathrm{Ge}$ lenkkörper bei intermittierenden Blockierungen das Auftreten von sekundären Knorpelläsionen mit der Gefahr einer Früharthrose. Die Prävention posttraumatischer Fehlstellungen und Inkongruenzen durch frühzeitige und adäquate Frakturversorgung ist eine wesentliche Behandlungsstrategie zur Vermeidung einer intrinsischen Kontraktur.

\section{Extrinsische Kontrakturen}

Den extrinsischen Kontrakturen des Ellbogengelenks liegen reversible oder irreversible Veränderungen des paraartikulären Bindegewebes zugrunde. Diese betreffen am häufigsten die Gelenkkapsel und den Seitenbandapparat; seltener sind Kontrakturen des Muskel- und Sehnengewebes oder der Haut. Eine Sonderstellung nehmen die ektopen paraartikulären Ossifikationen ein, deren Pathogenese noch weitgehend ungeklärt ist. Daneben können extraartikuläre knöcherne Fehlstellungen zu einer erheblichen Bewegungseinschränkung führen.

\section{Kapsuläre Kontraktur}

Kapsuläre Fibrosen im Kindesalter treten als Folge von Immobilisation nach suprakondylären Humerusfrakturen und v. a. nach Luxationen des Ellbogengelenks auf. Auch im Erwachsenenalter spielt die Länge der Ruhigstellung nach Trauma für die Entstehung posttraumatischer Kapselfibrosen eine Rolle. Die genaue Inzidenz lässt sich nicht festlegen, da sie sowohl von der Schwere der Verletzung als auch von der Art und der Dauer der Ruhigstellung abhängig zu sein scheint. Pathomorphologisches Substrat der kapsulären Steife ist eine Fibrosierung der Gelenkkapsel mit Verdickung und intraartikulärer Bridenbildung.

Die kapsulären Kontrakturen sind häufig innerhalb mehrerer Monate reversibel und sollten daher nicht frühzeitig zu Manipulationen oder chirurgischen Eingriffen Anlass geben. Vor allem bei posttraumatischen Ellbogensteifen im Wachstumsalter ist die Indikation $\mathrm{zu}$ chirurgischen Maßnahmen wegen der günstigen Rückbildungstendenz zurückhaltend $\mathrm{zu}$ stellen.

Besteht eine Bewegungseinschränkung länger als 2 Monate, sind neben aktiven auch passive Bewegungs- und Dehnungsübungen, evtl. in Kombination mit einer Schienenbehandlung, indiziert. Schwellung, Schmerz und Entzündungszeichen müssen dabei sorgfältig beachtet werden, da eine zu aggressive Therapie die Kontraktur verschlimmern kann.

Die geschlossene Arthrolyse mit einem Distraktionsfixateur stellt einen relativ neuen, semiinvasiven Behandlungsansatz dar [14].

Bei zunehmender Bewegungseinschränkung oder über 6 Monate ausbleibender Besserung der Kontraktur stellen Kapselrelease und -exzision, endoskopisch oder über einen lateralen, medialen oder kombinierten Zugang ausgeführt, ein Verfahren mit deutlicher Funktionsverbesserung dar. Dabei sind die endoskopischen Verfahren wegen dem relativ hohen Komplikationsrisiko durch die Gefährdung neurovaskulärer Strukturen und einer eingeschränkten Bewegungsverbesserung leichteren Fällen und geübten Endoskopikern vorbehalten [5, 12, 17]. Mansat u. Morrey [7] berichteten bei einer Nachuntersuchung mehr als 3 Jahre nach einer „lateral column procedure" mit kompletter Kapselexzision über einen radialen Zugang von einer Steigerung des Flexionsbogens um durchschnittlich $43^{\circ}$. Bei $26 \%$ der Patienten verschlechterte sich allerdings die Beweglichkeit in Folge des operativen Eingriffs.

\section{Ossäre Kontraktur}

Beim jüngeren Patienten sind posttraumatische Schäden in Folge von Frakturen mit Beteiligung der Epiphysenfuge im Kindes- und Jugendalter am häufigsten. Das daraus resultierende extraartikuläre Fehlwachstum kann zu einer Achsdeformität mit funktioneller Bewegungseinschränkung (z. B. Beugehemmung bei Rekurvations-, Streckhemmung bei Antekurvationsfehlstellung) führen. Pseudarthrosen der Epikondylen führen zur Bewegungseinschränkung und Instabilität. Die posttraumatische Fehlstellung des distalen Humerusendes stellt nach Abschluss der Frakturheilung und bei älteren Jugendlichen nach Wachstumsabschluss eine Indikation zur Korrekturosteotomie dar, die zu einer Verbesserung der funktionellen Beweglichkeit führt $[8,9]$. Bei Kindern unter dem 10. Lebensjahr sollte der Eingriff von einer engmaschigen Verlaufskontrolle abhängig gemacht werden; einerseits besteht im Wachstum ein Ausgleichspotenzial, andererseits kann bei progredienter Kontraktur die sekundäre Verkürzung der neurovaskulären Strukturen die Korrekturmöglichkeiten limitieren. Auch im Erwachsenenalter können fehlverheilte Frakturen des distalen Humerus oder des proximalen Ulnaendes zu erheblichen Bewegungseinschränkungen führen (Abb. 2). Bei der Arthrose im proximalen Radioulnargelenk führt die Radiusköpfchenresektion mit oder ohne endoprothetischem Ersatz zu einer Verbesserung der Beweglichkeit.

\section{Muskuläre Kontraktur}

Posttraumatisch v. a. nach Luxationen erworbene muskuläre Kontrakturen sind auf Einblutungen in den M. brachialis mit sekundärer Narbenformation und/ oder einer Myositis ossificans zurückzuführen. Die Volkmann-Kontraktur ist Folge eines ischämischen Kompartmentsyndroms der Unterarmmuskulatur als seltene Komplikation nach suprakondylären oder Unterarmfrakturen. Sie führt durch Muskelnekrosen zu einer komplexen Bewegungsstörung von Ellbogen- und Handgelenk.

\section{Kutane Kontraktur}

Ausgedehnte Weichteilwunden oder beugeseitige Verbrennungen können durch 

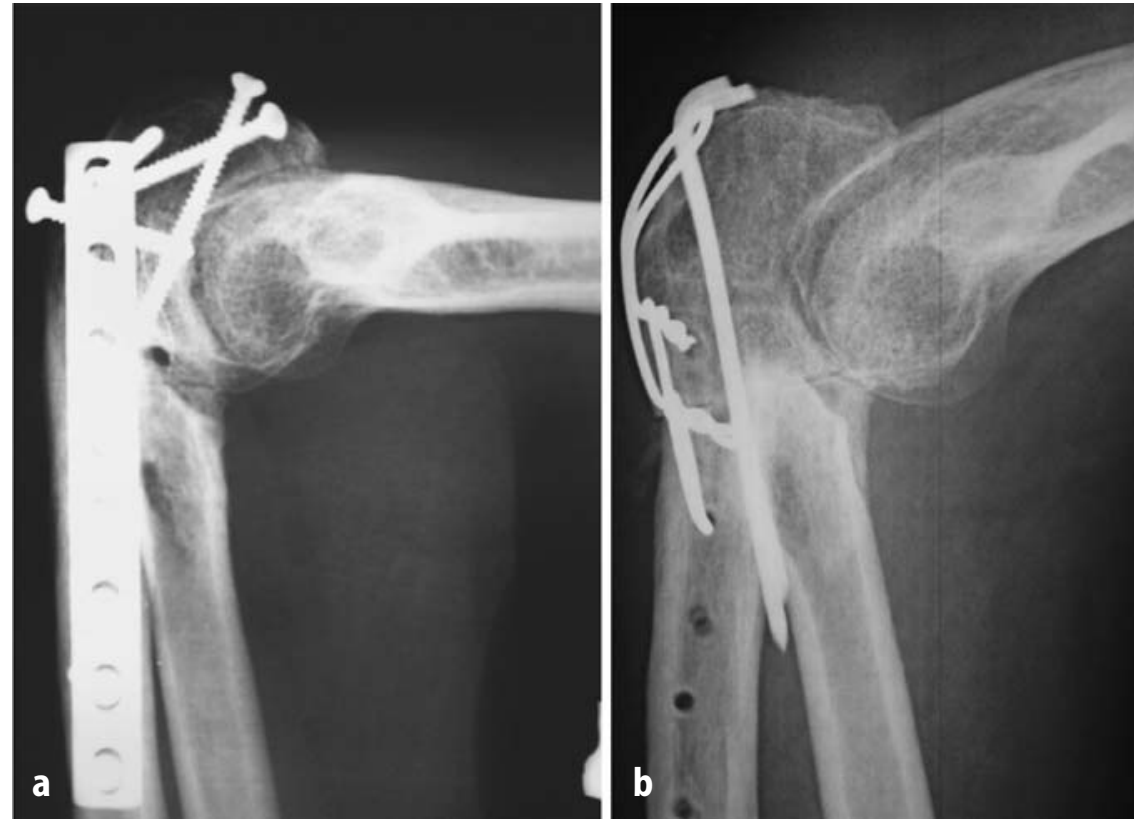

Abb. $2 a, b>$ Posttraumatische Ellbogenkontraktur (Extension-Flexion 0-90-110 ${ }^{\circ}$ ) bei fehlverheilter Olekranonfraktur, a präoperativ, b nach Korrekturosteotomie des Akromions (Extension-Flexion $\left.0-40-100^{\circ}\right)$

kutane Narbenbildung und Verklebungen mit den tiefer liegenden Strukturen $\mathrm{zu}$ neurovaskulären Störungen und $\mathrm{zu}$ einem ausgeprägten Streckdefizit führen.

\section{Ektope Ossifikationen}

Ektope Ossifikationen sind Verknöcherungen im Bereich des paraartikulären Bindegewebes, v. a. der Gelenkkapsel, der Kollateralbänder und der Muskulatur, infolge sehr unterschiedlicher lokaler und systemischer Noxen [13, 18]. Der Pathomechanismus der Entstehung dieser Ossifikationen, die histologisch identisch wie normaler Knochen trabekulär strukturiert und mineralisiert sind und deren Knochenmatrix bildende Zellen hochorganisierte Havers-Systeme produzieren, ist unbekannt. Die heterogenen posttraumatischen Umstände, unter denen vermehrt paraartikuläre Verknöcherungen beobachtet werden, sind:

\section{- Extremitäten- und Gelenktrauma}

- Gedecktes Schädel-Hirn- und Rückenmarktrauma

- Verbrennungen.

Nach traumatischen Luxationen des Ellbogengelenks, v. a. in Kombination mit gelenknahen knöchernen Verletzungen
(Abriss des Processus coronoideus, knöcherne Bandausrisse, Radiusköpfchenfraktur) kommt es in etwa 20\% der Fälle zu paraartikulären Ossifikationen bevorzugt im Bereich der ventralen oder dorsalen Kapsel. Diese führen lageabhängig zu einer unterschiedlich ausgeprägten Einschränkung des Flexionsbogens. Frakturen des proximalen Unterarms, Rupturen der distalen Bizepssehne v. a. nach chirurgischer Refixation und andere Weichteilverletzungen können außerdem zu einer radioulnaren Synostose mit Einschränkung oder Aufhebung der Pro- und Supinationsbewegung führen. Verknöcherungen werden gehäuft nach wiederholten traumatisiedylären Frakturen im Kindesalter [16] oder nach aggressiver passiver frühfunktioneller Behandlung bei Ellbogenverletzungen und nach geschlossenen Manipulationen in Narkose bei vorbestehender Gelenksteife beobachtet.

In der Folge von gedeckten Verletzungen des ZNS kommt es in $4 \%$ der Fälle zu paraartikulären Verknöcherungen im Bereich des Ellbogens, am häufigsten posterolateral beginnend mit im Extremfall vollständiger Ankylosierung die Kongruenz der Gelenkflächen meistens erhalten, während eine brückenförrenden Repositionsmanövern bei kondes Gelenks [3]. In dieser Situation ist mige Verknöcherung der Kapsel zu beobachten ist.

Nach höhergradigen Verbrennungen werden in etwa $1 \%$ der Fälle ellbogennahe Verknöcherungen beschrieben [2]. Diese betreffen bei unveränderter Gelenkfläche häufig die paraartikuläre Muskulatur im Sinn einer Myositis ossificans. Qualität und Ausmaß der Bewegungseinschränkung sind vom Muster der befallenen Muskulatur abhängig. Indikation, Zeitpunkt und Technik der chirurgischen Intervention bei paraartikulären Verknöcherungen werden kontrovers diskutiert. Die ${ }^{99} \mathrm{Tc}-K n o c h e n-$ szintigraphie ist in der Formationsphase der Knochenbildung signifikant positiv; die Aktivität kann jedoch über Monate persistieren [10], sodass diese Untersuchung für die Operationsplanung von nur eingeschränkter Bedeutung ist. Gleiches gilt für den prädiktiven Wert der alkalischen Serumphosphatase, deren Erhöhung ein unsicheres Zeichen für die Aktivität der Verknöcherungen ist. Morrey [10] empfahl, die Ossifikationen radiologisch ausreifen zu lassen und 9-12 Monate nach ihrem Auftreten operativ $\mathrm{zu}$ intervenieren. Bei der chirurgischen Abtragung der Verknöcherungen sind ein atraumatisches Vorgehen mit sorgfältiger Blutstillung und Vermeidung der Verbreitung von Knochenmehl durch ausreichende Wundspülung sowie eine postoperative Kompressionsbehandlung eine essenzielle Rezidivprophylaxe. Radiologische Nachbestrahlung oder orale medikamentöse Therapie mit nichtsteroidalen Antiphlogistika bzw. Bisphosphonaten verringern das Rezidivrisiko.

\section{Literatur}

1. Cohen MS, Hastings H (1998) Post-traumatic contracture of the elbow. Operative release using a lateral collateral ligament sparing approach.J Bone Joint Surg Br 80-B:805-812

2. Evans $E B$ (1991) Heterotopic bone formation in thermal burns. Clin Orthop 263:94-103

3. Garland DE, O'Halloren RM (1982) Fractures and dislocations about the elbow in the head injured adult. Clin Orthop 168:38-44

4. Hertel R, Pisan M, Lambert S, Ballmer F (1997) Operative management of the stiff elbow: sequential arthrolysis based on a transhumeral approach.J Shoulder Elbow Surg 6:82-88

5. Jones GS, Savoie FH (1993) Arthroscopic capsular release of flexion contractures (arthrofibrosis) of the elbow. Arthroscopy 9:277-283 
6. Loew M (2001) Die Ellenbogenkontraktur ätiologische Übersicht und allgemeine Behandlungskonzepte. Orthopäde 30:587-592

7. Mansat P, Morrey BF (1998) The column procedure: a limited lateral approach for extrinsic contracture of the elbow.J Bone Joint Surg Am 80-A: 1603-1615

8. Marti RK, Ochsner PE, Bernoski FP (1981) Korrekturosteotomie des distalen Humerus beim Erwachsenen. Orthopäde 10:311-315

9. Marti R, Engelhardt P, Jakob R (1981) Behandlungsrichtlinien für distale intra- und extraartikuläre Humeruspseudarthrosen. Orthopäde 10:316-322
10. Morrey BF (2000) Ectopic ossification about the elbow. In: Morrey BF (ed) The elbow and its disorders. Saunders, Philadelphia, pp 437-446

11. Morrey BF, Kai-Nan A (2000) Functional evaluation of the elbow. In:Morrey BF (ed) The elbow and its disorders. Saunders, Philadelphia, pp 74-83

12. O'Driscoll SW, Morrey BF (1992) Arthroscopy of the elbow: diagnostic and therapeutic benefits and hasards.J Bone Joint Surg Am 74-A:84-91

13. Orzel JA, Rudd TG (1985) Heterotopic bone formation: clinical, laboratory and imaging correlation. J Nucl Med 26: 125-134

14. Pennig D, Gausepohl T, Mader K, Wolfgraten B (2001) Die Distraktionsarthrolyse mit dem humeroulnaren Bewegungsfixateur zur Behandlung der posttraumatischen Ellenbogensteife. Orthopäde 30:635-644
15. Pötzl W, Ritsch M, Bakemeier EM, Steinbeck J (2000) Ergebnisse nach operativer Therapie bewegungseingeschränkter Ellenbogengelenke.Z Orthop Ihre Grenzgeb 138: 118-122

16. Stans AA, Morrey BF (2000) Post-traumatic elbow stiffness in children. In:Morrey BF (ed) The elbow and its disorders. Saunders, Philadelphia, pp 287-292

17. Strobel MJ, Eckardt OA, Eichhorn HJ (2001) Die arthroskopische Therapie bei Bewegungseinschränkungen des Ellenbogengelenks. Orthopäde 30:610-618

18. Thompson HG, Garcia A (1967) Myositis ossificans: aftermath of elbow injuries. Clin Orthop 50:129-137 